\title{
Glioblastoma and Colorectal Adenocarcinoma in an Adolescent Girl with Constitutional Mismatch Repair Deficiency Syndrome Mimicking Neurofibromatosis Type-I
}

\begin{tabular}{|c|c|}
\hline Author(s) & 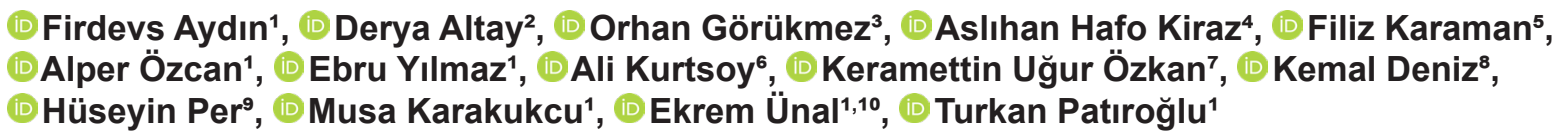 \\
\hline Affiliation(s) & $\begin{array}{l}\text { 1Division of Pediatric Hematology and Oncology, Department of Pediatrics, Erciyes University, Faculty of Medicine, Kayseri, Turkey } \\
\text { 2Division of Pediatric Gastroenterology, Department of Pediatrics, Erciyes University, Faculty of Medicine, Kayseri, Turkey } \\
\text { 'Department of Medical Genetics, Health Sciences University, Bursa Training and Research Hospital, Bursa Turkey. } \\
\text { 'Department of Medical Genetics, Health Sciences University, Kayseri Training and Research Hospital, Kayseri, Turkey. } \\
\text { 5Division of Pediatric Radiology, Department of Radiology, Erciyes University, Faculty of Medicine, Kayseri, Turkey } \\
\text { 'Department of Neurosurgery, Erciyes University, Faculty of Medicine, Kayseri, Turkey } \\
\text { 'Division of Pediatric Surgery, Erciyes University, Faculty of Medicine, Kayseri, Turkey } \\
\text { 'Department of Pathology, Erciyes University, Faculty of Medicine, Kayseri, Turkey } \\
\text { 9Division of Pediatric Neurology, Department of Pediatrics, Erciyes University, Faculty of Medicine, Kayseri, Turkey } \\
\text { 10Department of Molecular Biology and Genetics, Gevher Nesibe Genom and Stem Cell Institute, Genome and Stem Cell Center (GENKOK), } \\
\text { Erciyes University, Kayseri, Turkey }\end{array}$ \\
\hline $\begin{array}{c}\text { Article } \\
\text { Information }\end{array}$ & $\begin{array}{l}\text { Article Type: Case Report } \\
\text { Article Group: Child and Adolescent Psychiatry }\end{array}$ \\
\hline
\end{tabular}

Cite this article as: Aydın F, Altay D, Görükmez O, Hafo Kiraz A, Karaman F, Özcan A, Yılmaz E, Kurtsoy, A, Özkan KU, Deniz K, Per H, Karakukcu M, Ünal E, Patıroğlu T. Glioblastoma and Colorectal Adenocarcinoma in an Adolescent Girl with Constitutional Mismatch Repair Deficiency Syndrome Mimicking Neurofibromatosis Type-I. J Pediatr Acad 2020; 1(1): 34-38.

\section{Abstract}

Children with constitutional mismatch repair deficiency syndrome (CMMRDS) are prone to different types of cancers. A 16-year-old girl who was misdiagnosed with neurofibromatosis Type-I (NF-I) for 1 years had experienced glioblastoma and colonic adenocarcinoma. After operation, chemotherapy and radiotherapy were started for adenocarcinoma. Genetic analysis from the patient, effected brother, and mother showed heterozygote (c. $479+36 \mathrm{~A}>\mathrm{G})$ mutation in the intron 4 region of NF-1 gene. Initially, it was thought that this genetic variant was causative. Furthermore, next generation sequencing showed that the index patient and his brother have homozygote (c.1444 C>T) mutations in the MSH6 gene which are associated with CMMRDS both died because of colonic adenocarcinoma, and T cell non-Hodgkin lymphoma, respectively. Patients with CMMRDS may resemble NF-I. The physicians must not be confused with the previous diagnosis. Increased awareness of CMMRDS, and prompt evaluation for an underlying genetic background is advised if there are unexpected cancer in patients with NF-I.

Keywords: : Adenocarcinoma, adolescent, DNA mismatch repair, neurofibromatosis type 1

Correspondence: Ekrem Ünal, Erciyes University, Faculty of Medicine, Department of Pediatrics, Division of Pediatric Hematology and Oncology, 38030, Melikgazi , Kayseri, Turkey --- Department of Molecular Biology and Genetics, Gevher Nesibe Genom and Stem Cell Institution, Genome and Stem Cell Center (GENKOK), Erciyes University, Kayseri, Turkey

E-mail: drekremunal@yahoo.com.tr / ekremunal@erciyes.edu.tr 


\section{Introduction}

Neurofibromatosis type 1 (NF-I) is an autosomal dominant disorder with multiple system involvement, and characterized by increased incidence of both benign and malignant tumors. NF-I occurs with an estimated incidence of 1 in 2500 to 3000 individuals independent of ethnicity, race, and gender. ${ }^{1}$ Although cancer can develop at any age, the type of tumors varies in adults and children. NF-I is common in children with optic nerve glioma, and brain tumors. ${ }^{2}$ The constitutional mismatch repair deficiency syndrome (CMMRDS) is a rare condition characterized by hematologic malignancies, brain tumors and cancers associated with Lynch syndrome in childhood. ${ }^{3}$ Many patients with CMMRDS have café au lait spots (CLS) and similiar features of NF-I such as cancers. $^{3}$

Colorectal cancers are very rare in childhood and are usually associated with cancer susceptibility syndromes. ${ }^{4-6}$

Here we present a case with CMMRDS mutation detected by genetic tests after the development of colon adenocarcinoma and glioblastoma in an adolescent girl followed with the misdiagnosis of NF-I. We discuss her clinical and genetic features based on the reported case series.

\section{Case}

A 16-year-old girl with no previous disease was admitted to the pediatric emergency of Erciyes University Hospital with seizure. The patient's parents are firstdegree cousins. There was neither significant feature in the patient's medical history, nor a familial history of cancer. She has 4 siblings, and her 12-year-old brother has about 15 CLS. But her brother does not have any disease history.

She had more than 15 CLS in different parts of her body on physical examination. Ophthalmologic examination was negative for Lisch's nodules. Pathological features were not seen in other system examinations. Computerized tomography was performed because of head trauma to the patient. The mass in the right frontoparietal region of the patient (Figure 1a, 1b) was operated. The pathology examination was consistent with glioblastoma Figure 1c, $1 \mathrm{~d}, 1 \mathrm{e})$. She received radiotherapy for 1 month and then received temozolomide at a dose of $200 \mathrm{mg} / \mathrm{m}^{2}$. Antiepileptic drugs (clobazam, levetiracetam and phenytoin) were also started because of refractory convulsions.

In the follow-up period, the patient was admitted to the emergency department with severe abdominal pain one year later. The radiological images showed stenotic colonic segment with wall thickening (Figure 2a). She was operated because of colon perforation. During this operation, stricture of colonic segment is observed. In colonoscopy, two polyps, approximately $4 \times 3 \mathrm{~cm}$ in size, were observed in the rectum and a $3 \times 2 \mathrm{~cm}$ polyp was observed at approximately $20 \mathrm{~cm}$ from the anal verge. Mucosa of the rest of the colon was normal. The pathology report of the patient was relevant with the adenocarcinoma (Figure $\mathbf{2 b}$ ). The patient was reoperated and a large portion of the colon was removed and colostomy was opened. The genetic examination from patient, and the family (mother, father, and 3-yearsold brother) were done. It was also noticed that her mother and her brother had CLS. Genetic examination revealed a heterozygous (c. $479+36 \mathrm{~A}>\mathrm{G})$ mutation in the intron 4 region of the NF-1 gene (dbSNP: rs1370379787). Her mother and brother also had the same mutation.

NF1 findings and synchronous tumors at different sites suggested CMMRDS. We applied clinical exome sequencing and Sanger sequencing for the patient. Genomic DNAs were extracted from peripheral venous blood using the QIAamp® DNA Mini Kit (QIAGEN, Ankara, Turkey). The Clinical Exome Solution (SOPHiA GENETICS, Switzerland) was used to exome enrichment. All procedures were carried out according to the manufacturer's protocols. It is a capture-based target enrichment kit and covers 4,493 genes with known inherited disease causing mutations. Paired-end sequencing was performed on an Illumina NextSeq 500 system (Illumina, San Diego, California, USA) with a read length of $150 \times 2$. Base calling and image analysis were conducted using Illumina's Real-Time Analysis (Integrated to NextSeq 500 system) software. The BCL (base calls) binary is converted into FASTQ utilizing Illumina package bcl2fastq. All bioinformatics analysis performed on Sophia DDMTM platform (SOPHiA GENETICS SA., Switzerland), which includes algorithms for alignment and calling single nucleotide polymorphisms (SNPs) and small indels (Pepper, SOPHiA GENETICS' patented algorithm), calling copy number variations (Muskat, SOPHiA GENETICS' patented algorithm) and functional annotation (Moka, SOPHiA GENETICS' patented algorithm). Raw reads were aligned to the human reference genome (GRCh37/hg19). Variant filtering and interpretation performed on Sophia DDMTM. Integrative Genomics Viewer (IGV) was used to bam file visualization (7). Next generation sequencing (NGS) showed a homozygous nonsense mutation, c.1444C>T, p.(Arg482*) in MSH6 (NM_000179) gene. The mutation was confirmed by the Sanger sequencing. Sanger sequencing was also applied to relatives of the patient. The mutation was homozygous for the patient and patient's younger brother, while heterozygous for other family members (Figure 3).

The patient was given a chemotherapy course containing oxaliplatin, 5 fluorouracil, and calcium leucovorin (folinic acid) for adenocarcinoma in the colon. In the continuation of the treatment radiotherapy was started. After radiotherapy. The general condition of the patient was improved with these treatments. But just after three months, adenocarcinoma recurred in the colostomy site, so nivolumab treatment was started. The patient was re-operated but the tumor could not be completely resected. The tumor continued to grow at the site of colostomy. She died after two months of the diagnosis of adenocarcinoma.

After the detection of mutation at the MSH6 gene, sibling with the homozygous mutation was also examined. Brain magnetic resonance imaging revealed a mass in the frontal region. Colonoscopy revealed polyps with tubulovillous adenoma. Nivolumab treatment was advised but the family refused. Two months later, the 

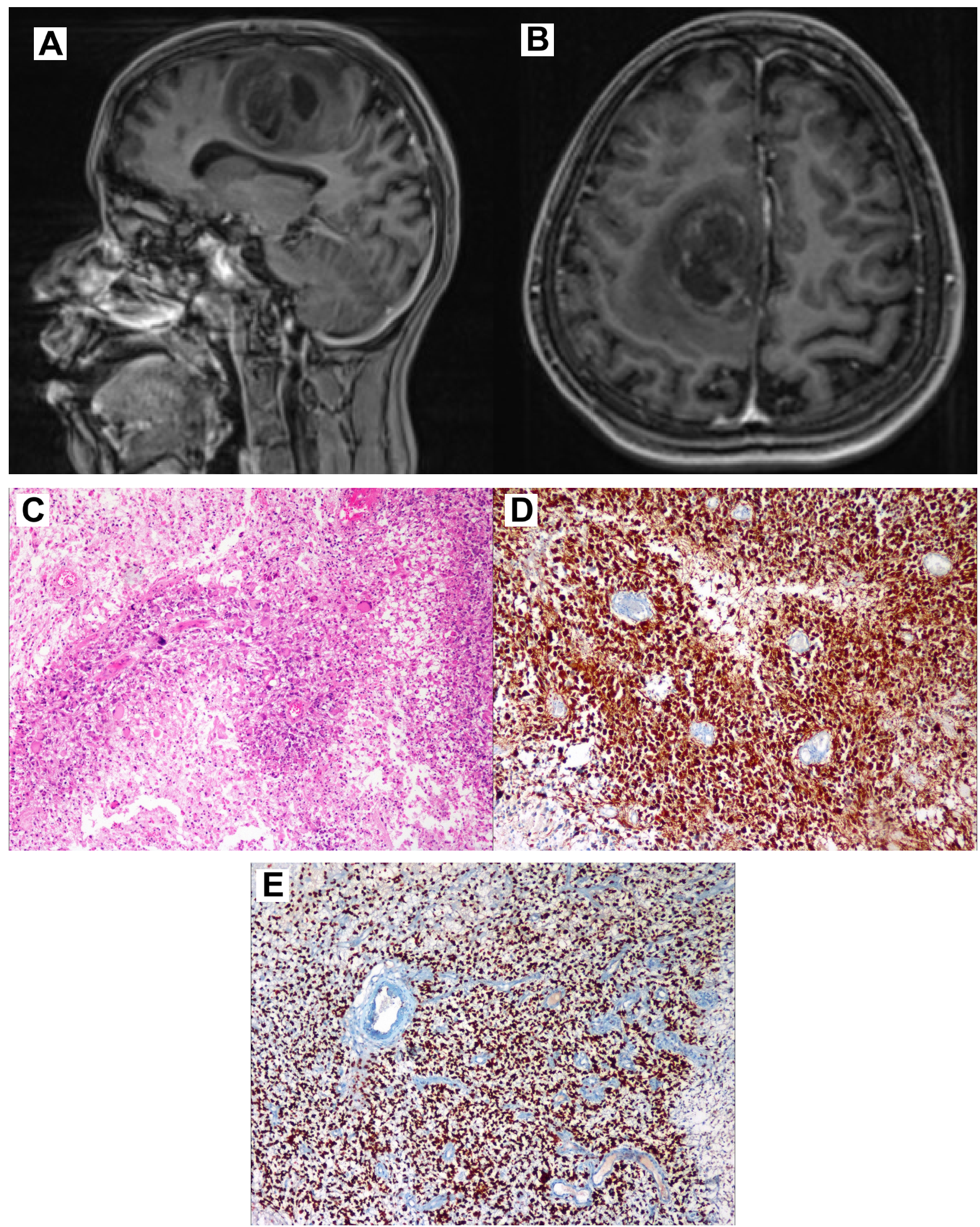

Figure 1. A heterogeneous cystic-necrotic mass is seen at right cerebral hemisphere vertex on sagittal (a), and axial (b) T1 Weighted MRI, with gadolinium contrast. Peripheral contrast enhancement is seen. Light microscopic image showed pleomorphic tumor cells with palisaded necrosis (Hematoxylin \& Eosin, x100) (c), Tumor cells displayed glial fibrillary acidic protein positivity (x100) (d), p53 positivity (x100) (e).

male patient presented with respiratory distress. The mass in the mediastinum was compressing the trachea. The patient was intubated and hospitalized in pediatric intensive care unit. The pathology result of the biopsy was reported as T cell non Hodgkin lymphoma. Steroid treatment was started but his condition worsened and he died within a month. 


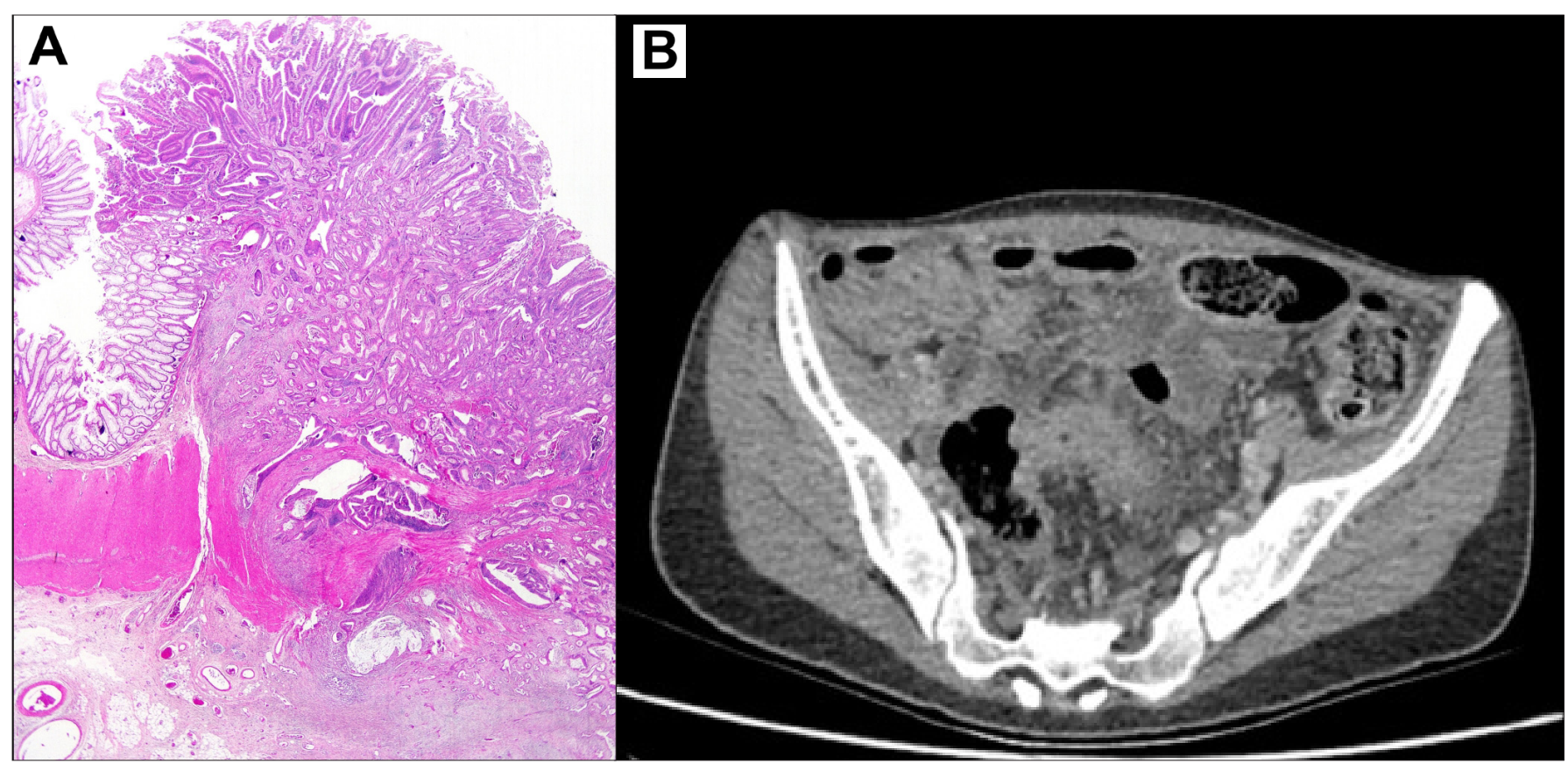

Figure 2. Sections form the colonic tumor showed neoplastic glands infiltrating the colonic wall through the serosa (Hematoxylin \& Eosin $x 100)$ (a). Stenotic colonic segment with wall thickening is seen in sigmoid on axial image of pelvic contrast enhanced computed tomography (b).

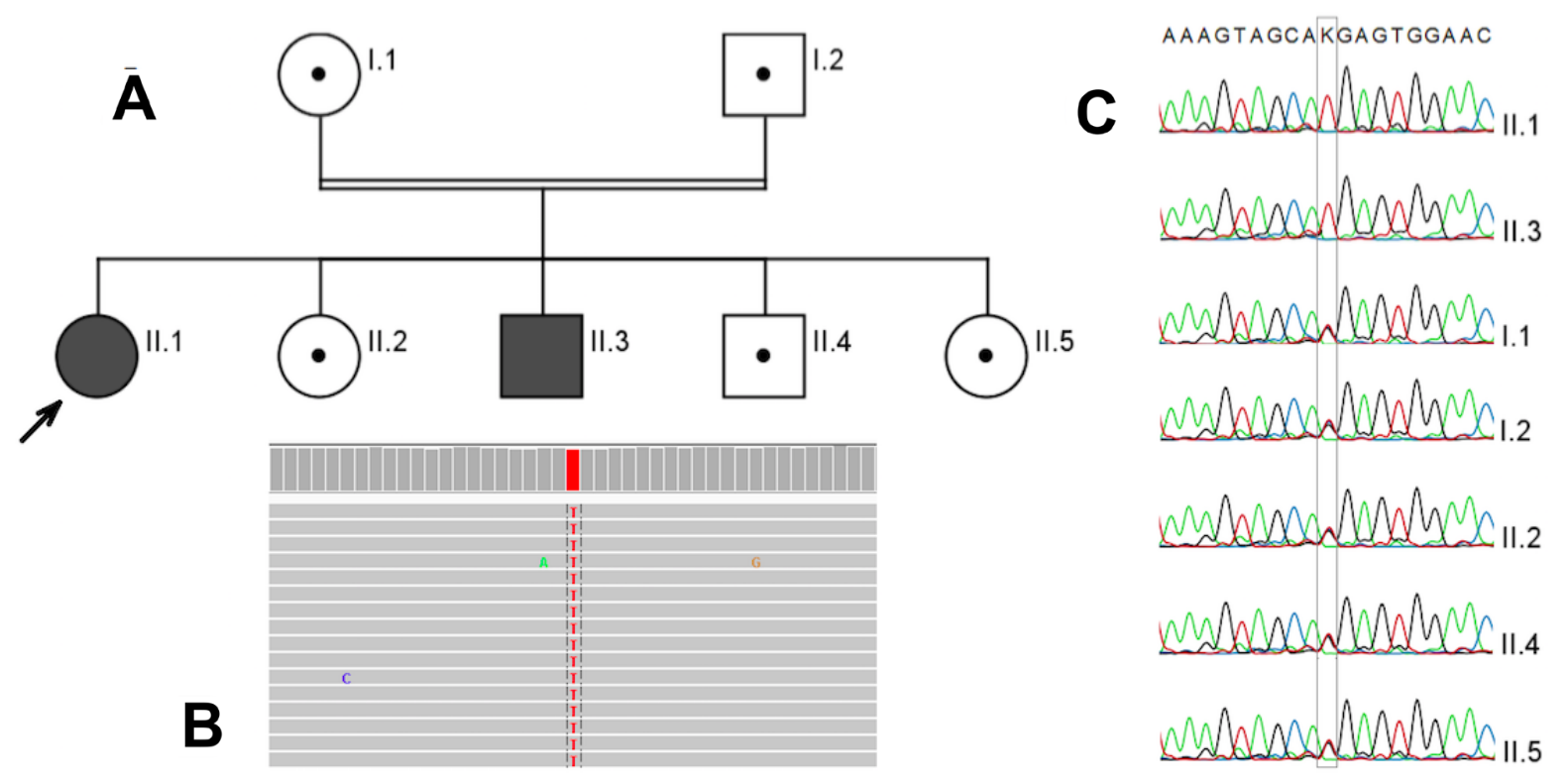

Figure 3. Pedigree of the family showing the identified MSH6 mutation (c.1444C>T, p.Arg482*). The arrow indicates the proband. Black symbols indicate the affected individuals. The proband, and II.3 are homozygous; I.1, I.2, II.2, II.4, and II.5 are heterozygous for the mutation (a). Excerpt of next-generation sequencing data visualized using Integrative Genomics Viewer (b). Results of DNA sequencing. A nonsense germline mutation, c.1444C>T, p.Arg482* in MSH6 (NM_000179) gene of the family (indicated by grey frame) (c).

\section{Discussion}

Constitutional mismatch repair (MMR) deficiency syndrome is a childhood cancer predisposition syndrome resulting from biallelic germline mutations in one of the four MMR genes (MLH1, MSH2, MSH6, or PMS2). The mostly encountered mutations in children with CMMRDS are PMS2 are MSH6 genes. ${ }^{8}$ Although both parents are compulsory carriers, family history may not always be observed. Therefore, it is not uncommon for affected children to have unaffected parents. ${ }^{9}$ In addition to the cancer predisposition, immune deficiencies and autoimmunity can be observed in children with CMMRDS. .,10 Our patients did not show any features of autoimmunity or immunodeficiency. The mortality rate among patients with CMMRDS is high and $29 \%$ of patients die of primary tumor. The median survival from the diagnosis of the first tumor in these patients is 27 months. ${ }^{11}$

Colorectal carcinomas are rare in the pediatric population and very few cases with NF-I have been reported. ${ }^{3,6,12,13}$ Changes in bowel habits and hematocesia are more common in left colon and rectum cancers. Iron deficiency anemia and tenesis can be seen in rectal tumors. Constipation and abdominal pain may be seen in all localization of colon cancer. .,13 $^{2}$

Trilling, and Faucheron ${ }^{14}$ performed a systematic review of the literature on acute intestinal obstruction due to 
NF-I by searching the major electronic data bases. They have identified 25 articles from 1972 to 2013 reporting 25 patients with NF-I who underwent laparotomy for acute intestinal obstruction. The acute intestinal obstruction in this study were classified as intrinsic obstruction, extrinsic obstruction, and intussusception. The underlying reasons were mainly due to neurofibroma, gastrointestinal stromal tumor, and adenocarcinoma. Only the histological examination of 4 of these 25 showed adenocarcinoma; none of the 4 reported cases of adenocarcinoma with NF-I in this study were in pediatric age group. ${ }^{14}$ Our patient was presented with abdominal pain and then bowel perforation. Because gastrointestinal carcinomas are documented in children with NF-I, the diagnosis, rapid evaluation of malignancy is necessary in the presence of gastrointestinal symptoms in these children. Different types of synchronous or meta-synchronous malignancies can be seen in children with CMMRS. ${ }^{3}$ Our patient had different types of malignancy that occurred synchronously.

Consanguineous marriage rate varies by countries. Homozygous cases of CMMRDS are more observed in countries such as Turkey. Whereas CMMRDS are generally associated with a combined heterozygous mutation in non-consanguineous families especially in Europe. ${ }^{15}$ The parents of our patient were relatives, but neither of them had cancer although both of them have heterozygous mutations. Only two of the five children had a homozygous mutation. One is our patient and the other is her brother, who has CLS on his body.

In conclusion, when rare malignancies associated with NF-I are detected, CMMRDS and other malignancyrelated genetic diseases should be excluded in children who have previously been misdiagnosed with NF-I. These children and their families should be closely monitored for malignancies, and genetic counseling must be considered.

Acknowledgments: The authors thank to Prof. Dr. Abdulhakim Coşkun, Prof. Dr. Duran Arslan, and Prof Dr. Halit Canatan for critical review of this manuscript.

Informed Consent: Written informed consent was obtained from patients who participated in this study.

Peer-review: Externally peer-reviewed.

Author Contributions: All of the authors declare that they have all participated in the design, execution, and analysis of the paper, and that they have approved the final version
Conflict of Interest: The authors have no conflict of interest to declare.

Financial Disclosure: The authors declared that this study has received no financial support

\section{References}

1. Varan A, Şen $H$, Aydın B, et al. Neurofibromatosis type 1 and malignancy in childhood. Clin Genet. 2016; 89: 341-345. [CrossRef]

2. Peltonen S, Kallionpää RA, Rantanen $M$, et al. Pediatric malignancies in neurofibromatosis type 1: A population-based cohort study. Int J Cancer. 2019; 145: 2926-2932. [CrossRef]

3. Wimmer K, Etzler J. Constitutional mismatch repair-deficiency syndrome: have we so far seen only the tip of an iceberg? Hum Genet. 2008; 124: 105-122. [CrossRef]

4. Urganci N, Genc DB, Kose G, et al. Colorectal Cancer due to Constitutional Mismatch Repair Deficiency Mimicking Neurofibromatosis I. Pediatrics. 2015; 136: e1047-1050. [CrossRef]

5. Kim SE, Heo EP, Yoon TJ, et al. Segmentally distributed neurofibromatosis associated with adenocarcinoma of the colon. J Dermatol. 2002; 29: 350-353. [CrossRef]

6. Ladd AP, Grosfeld JL. Gastrointestinal tumors in children and adolescents. Semin Pediatr Surg. 2006; 15: 37-47. [CrossRef]

7. Robinson JT, Thorvaldsdóttir H, Winckler W, et al. Integrative genomics viewer. Nat Biotechnol. 2011; 29: 24-26. [CrossRef]

8. Wimmer K, Kratz CP, Vasen HF, et al. Diagnostic criteria for constitutional mismatch repair deficiency syndrome: suggestions of the European consortium 'care for CMMRD' (C4CMMRD). J Med Genet. 2014; 51: 355-365. [CrossRef]

9. Tabori U, Hansford JR, Achatz MI, et al. Clinical management and tumor surveillance recommendations of inherited mismatch repair deficiency in childhood. Clin Cancer Res. 2017;23:e32e37. [CrossRef]

10. Tesch VK, IJspeert $H$, Raicht A, et al. No Overt Clinical Immunodeficiency Despite Immune Biological Abnormalities in Patients With Constitutional Mismatch Repair Deficiency. Front Immunol. 2018; 9: 1506. [CrossRef]

11. Lavoine N, Colas C, Muleris M, et al. Constitutional mismatch repair deficiency syndrome: clinical description in a French cohort. J Med Genet. 2015; 52: 770-778. [CrossRef]

12. Wimmer K, Rosenbaum T, Messiaen L. Connections between constitutional mismatch repair deficiency syndrome and neurofibromatosis type 1. Clin Genet. 2017; 91: 507-519. [CrossRef]

13. Ozaydın S, Atas E, Tanriseven M, et al. Colorectal Cancer in Patients 30 Years Old and Younger: A 17-Year Experience. Erciyes Med J. 2019; 41: 62-68. [CrossRef]

14. Trilling B, Faucheron JL. Intestinal obstruction in von Recklinghausen's disease. Colorectal Dis. 2014; 16: 762-768. [CrossRef]

15. Vasen HF, Ghorbanoghli Z, Bourdeaut F, et al. EU-Consortium Care for CMMR-D (C4CMMR-D). Guidelines for surveillance of individuals with constitutional mismatch repair-deficiency proposed by the European Consortium "Care for CMMR-D" (C4CMMR-D). J Med Genet. 2014; 51: 283-293. [CrossRef] 